Cahiers « Mondes anciens »

ANCIENS

Histoire et anthropologie des mondes anciens

$14 \mid 2021$

Thalassotopies

\title{
Mondes en suspens
}

Poséidon et le basculement des îles vers l'histoire

Unsettled Worlds. Poseidon and the Swift of Islands towards History

\section{François de Polignac}

\section{(2) OpenEdition}

Journals

Édition électronique

URL : https://journals.openedition.org/mondesanciens/3395

DOI : $10.4000 /$ mondesanciens.3395

ISSN : 2107-0199

Éditeur

UMR 8210 Anthropologie et Histoire des Mondes Antiques

Référence électronique

François de Polignac, " Mondes en suspens », Cahiers « Mondes anciens » [En ligne], 14 | 2021, mis en ligne le 06 septembre 2021, consulté le 21 janvier 2022. URL : http://journals.openedition.org/ mondesanciens/3395; DOI : https://doi.org/10.4000/mondesanciens.3395

Ce document a été généré automatiquement le 21 janvier 2022.

\section{cc) (1) () $\odot$}

Les Cahiers «Mondes Anciens » sont mis à disposition selon les termes de la licence Creative Commons Attribution - Pas d'Utilisation Commerciale - Pas de Modification 4.0 International. 


\title{
Mondes en suspens
}

\author{
Poséidon et le basculement des îles vers l'histoire \\ Unsettled Worlds. Poseidon and the Swift of Islands towards History
}

\section{François de Polignac}

1 Dans le premier livre de ses Argonautiques, Apollonios de Rhodes consacre un long passage (v. 936-1152) aux aventures des héros de la nef Argô chez les Dolions, un peuple vivant en Propontide, sur le site de la future cité de Cyzique. Après avoir franchi l'Hellespont, les navigateurs atteignent une île montagneuse qui n'est séparée de la rive asiatique de la Propontide que par un passage étroit ${ }^{1}$. Sur cette île qui porte le nom de "Mont des ours ", vit une population de "nés de la terre ", gègénées, géants pourvus de six bras et qualifiés de féroces et sauvages, hubristai te kai agrioi (v. 942). À côté, dans la plaine au pied de la montagne et sur l'isthme, vit le peuple des Dolions qui sont tout le contraire de leurs voisins : accueillants et pacifiques, ils possèdent une ville et sont gouvernés par le roi Cyzicos, à peine sorti de l'adolescence, qui avec son épouse Cleitè accorde l'hospitalité aux Argonautes. Ainsi est dressé le tableau de l'opposition entre deux mondes radicalement différents, celui de créatures primordiales redoutables, analogues à bien d'autres figures monstrueuses issues des générations les plus archaïques de la genèse du monde, et celui d'une humanité déjà pourvue des traits de la civilisation; deux mondes, toutefois, qui, malgré leur différence de nature, vivent au voisinage immédiat l'un de l'autre. Car le dieu Poséidon rend possible la perpétuation de ce voisinage en neutralisant la menace qu'il pourrait faire peser sur les Dolions : ceux-ci sont les descendants du dieu qui les protège en empêchant les gègénées de les attaquer (v. 951-952).

Dans la perspective d'une lecture des Argonautiques comme «itinéraire à travers la sauvagerie» (Cusset 2004), le tableau que dresse Apollonios semble être un cas emblématique d'opposition entre un monde sauvage, celui de l'île et de la montagne peuplée de créatures primitives, et un monde civilisé, celui des Dolions vivant dans la plaine amène et accessible. Cette opposition n'est cependant pas statique. Jouant avec les différentes caractérisations d'un paysage littéraire très construit, l'épisode fait passer les Argonautes à travers toutes les étapes d'une action civilisatrice qui investit l'espace de nouvelles significations (André 2010). Dans cette optique, la protection que 
Poséidon octroie aux Dolions pourrait sembler s'inscrire dans un rejet du monde sauvage, une hostilité envers les géants aux six bras qui ferait des Dolions et de leur protecteur divin les champions d'une humanisation de la région aux dépens des êtres primordiaux qui la peuplaient à l'origine. Mais il faut dépasser cette vision binaire où Poséidon serait du seul côté de la civilisation. En effet, les «nés de la terre » du Mont des Ours appartiennent clairement à l'univers multiforme des créatures à la taille et à la force démesurées - comme les Cent-Bras avec qui ils partagent la multiplicité des membres supérieurs, ou encore les Cyclopes et tant d'autres personnages comme Pélôr (les « nés de la terre » sont qualifiés de "monstres terribles », aina pelôra, au vers 996), Orion, Aigaiôn, Alcyonée - que les légendes rapprochent tous de Poséidon et placent dans son entourage, sinon même dans sa progéniture (Vian 1944 et 1952). Toutes ces créatures assistent souvent le dieu dans ses œuvres telluriques de fracturation des continents, d'ouverture ou de fermeture de détroits et de passages: partout leur vigueur exceptionnelle accompagne les grands ébranlements par lesquels le dieu remodèle et appose sa marque sur le paysage (Polignac 2017). Mais en même temps, si leur hybris pousse ces créatures à la rébellion contre les dieux, Poséidon est aussi parmi les premiers à les combattre et à les punir de leur orgueil. Le dieu exerce en quelque sorte une forme de souveraineté, ou du moins de domination sur le monde multiforme de la monstruosité et de l'excès où l'on trouve bon nombre de ses propres enfants. De ce fait, même si d'après Apollonios qui utilise là un thème récurrent (Pirenne-Delforge et Pironti 2016, p. 264-270), Héra aurait nourri les géants de Cyzique pour en faire une des "épreuves d'Héraclès» (v. 996-997). C'est aussi à l'univers de Poséidon que ces êtres appartiennent, contrôlant ces êtres dangereux en exerçant sur eux une forme de maitrise. On peut donc considérer que c'est en vertu d'une forme de maîtrise générale que le dieu exerce sur ces créatures monstrueuses, et pas seulement en raison de sa qualité de géniteur des Dolions, que Poséidon maintient la paix entre les deux peuples. Cette paix paraît être un équilibre établi entre deux formes de vie, celle des êtres primordiaux et celle des humains, généralement inconciliables mais que Poséidon protège également et à proximité immédiate l'une de l'autre ${ }^{2}$. Ici comme ailleurs, pour reprendre une expression que j'ai déjà employée, le propre du dieu serait de «tenir ensemble le dissemblable » (Polignac 2017, p. 77). La coexistence pacifique qu'il garantit entre les géants aux six bras et les Dolions n'est pas sans évoquer une sorte d'âge d'or. Un lieu, où géants et humains vivent côte à côte sous la protection d'un dieu, ne peut appartenir à l'espace et au temps de l'histoire ordinaire. De ce fait, l'île s'inscrit dans un espace et un temps bien particuliers: un espace protégé des atteintes du monde extérieur et un temps en suspens, hors du cours de l'histoire.

C'est précisément cet équilibre que l'arrivée des Argonautes va rompre. Informé par un oracle qu'arriverait un jour une expédition de héros nés des dieux, le roi Cyzicos fait le meilleur accueil à Jason et à ses compagnons : sacrifices et banquet suivent ainsi leur débarquement (v. 961-984). Mais le lendemain, tandis que certains des Argonautes font l'ascension du mont Dindymon (montagne de l'île qui domine le Mont des Ours, comme le précise Strabon, XII, 8, 11) pour repérer leur route prochaine et que d'autres amènent le navire dans le "Port Clos", un des quatre ports de Cyzique, les gègénées descendus de la montagne commencent à lancer d'énormes rochers à l'entrée du port pour y bloquer Argô. Les Argonautes sont menacés d'enfermement dans un lieu devenu hostile, ce qui réduirait à néant toute leur entreprise ${ }^{3}$. Héraclès, resté à la tête des plus jeunes pour garder la nef, repousse les êtres sauvages de ses flèches; accourus à la rescousse, les Argonautes redescendus de la montagne achèvent la tâche en 
exterminant tous les «nés de la terre » dont les corps sont alignés tête-bêche sur la plage. Sans sépulture, dépourvus d'une identité individuelle reconnaissable, les géants vaincus sont étalés comme une sorte de pêche monstrueuse. L'épisode eût-il pris fin à cet endroit, le passage des Argonautes et l'intervention d'Héraclès pourraient être assimilés à une banale action civilisatrice : les héros auraient débarrassé les gentils Dolions de leurs affreux voisins et leur auraient ainsi assuré sécurité, paix et prospérité. La vie de la cité aurait semblé s'épanouir sur les cadavres des géants vaincus. Les Argonautes auraient alors pu repartir vers de nouvelles aventures, revêtus de la gloire d'avoir porté secours à un peuple menacé par la sauvagerie primitive.

Mais ce n'est pas ainsi que les choses se passent. Les Argonautes repartent en effet, mais à la nuit tombée, sont pris dans une tempête qui les rejette sur le rivage des Dolions, sans qu'ils reconnaissent les lieux ${ }^{4}$. De leur côté les Dolions ne reconnaissent pas les Argonautes et, croyant à une attaque nocturne, se mettent à les combattre. On notera en passant que, pour les uns comme pour les autres, la perte de toute identité renvoie au massacre indistinct des géants. Dans la bataille, le roi Cyzicos est tué par Jason, et nombre de valeureux Dolions tombent avec lui ; ce n'est qu'à l'aube que la méprise apparaît dans toute son étendue. Avec le jour l'identité reprend ses droits : durant les trois journées suivantes, Argonautes et Dolions célèbrent ensemble les funérailles du roi et de ses compagnons; même la reine Cleitè met fin à ses jours pour ne pas survivre à son époux. Après douze jours où les Argonautes restent bloqués par des vents contraires, Jason, sur le conseil du devin Mopsos, remonte sur le Dindymon avec ses compagnons pour sacrifier à la Mère des dieux; à la suite de quoi Argô peut reprendre la mer (v. 1078-1152). Le passage des Argonautes n'est donc pas un désastre pour les seuls «nés de la terre »: la mort de Cyzicos et Cleitè, sans descendance, marque aussi la fin de l'âge des Dolions et de son caractère exceptionnel tels qu'ils avaient été préservés jusque-là. L'extermination des géants préfigure le massacre des Dolions: l'une et l'autre sont indissociables et forment les deux faces d'une même réalité. Géants et Dolions forment en effet système et c'est bien l'ensemble du monde maintenu en équilibre par Poséidon avec sa double composante primordiale et humaine qui disparaît; c'est l'espèce d'âge d'or où se perpétuait ce monde en suspens qui est anéanti par l'arrivée du héros. Et ce basculement, jalonné par toute une série de gestes rituels, permet de faire entrer l'île dans une nouvelle ère, pleinement historique cette fois : celle de la cité de Cyzique, fondée par les Milésiens dans le premier quart du VII ${ }^{\mathrm{e}}$ siècle.

5 L'épisode en effet, comme toujours chez Apollonios, fourmille de références à des données topographiques et cultuelles historiques propres à la région concernée, qui y trouvent leur origine. À leur arrivée, les Argonautes ancrent leur navire dans l'un des ports de l'île, le "Beau Port " (Kalos Limèn) où ils procèdent à un changement d'ancre ; la pierre qu'ils laissent parce que trop petite est déposée près d'une source proche, la source Artakiès. Plus tard, ajoute Apollonios, les «Ioniens Néléides» (les Milésiens fondateurs de Cyzique) consacrèrent cette pierre dans le temple d'Athéna Jasonienne, en obéissance à un oracle d'Apollon (v. 953-960). Ensuite, accueillis par le roi Cyzicos, les Argonautes sont invités à pousser un peu plus loin jusqu'au " port de la ville »; là, ils élèvent près du rivage un autel à Apollon Ekbasios, "du débarquement", et sacrifient au dieu (v. 965-967). Le lieu où les héros abordent de nouveau le rivage des Dolions lors de la nuit de tempête est marqué par la pierre dite "sacrée " où ils attachent leurs amarres (v. 1018-1020). Apollonios donne ensuite les noms des Dolions (tués par les différents héros grecs lors de la bataille nocturne), et précise qu'il s'agit 
des guerriers "qu'aujourd'hui encore les habitants honorent comme des héros" (v. 1048) : c'est dans la mort que les Dolions acquièrent une pleine identité, en tant que marqueurs de l'espace et de l'histoire de la cité. La référence à un culte rendu sur un monument connu de la Cyzique historique est claire. Le roi Cyzicos, qui reçoit des funérailles dignes de l'épopée, est enseveli dans un tombeau «toujours visible pour la postérité » dans la plaine dite Leimônion (de la prairie) (v. 1057-1062). Le souvenir de la reine Cleitè est de son côté associé à une source que les Nymphes des bois font surgir avec les larmes qu'elles avaient versées pour elle (v. 1066-1069). Le culte rendu « encore maintenant», chaque année, aux deux défunts est caractérisé par des pratiques alimentaires particulières: en souvenir de la douleur des Dolions, si vive qu'ils n'avaient plus à cœur de moudre le grain et ne mangeaient plus que des aliments crus, les Ioniens de Cyzique utilisent les meules publiques, et non les leurs propres, pour fabriquer les galettes rituelles (v. 1070-1077). Rite et récit se rejoignent ici pour signifier la fin des Dolions en tant qu'hommes mangeurs de pain, ce qui souligne à nouveau que le cycle entamé par les destructions des «nés de la terre » n'aboutit en rien au triomphe de la civilisation. Enfin, les Argonautes instituent le culte de la Mère des dieux sur le mont Dindymon.

6 La venue des Argonautes, en même temps qu'elle met fin au monde ancien, sert ainsi à mettre en place le paysage cultuel et monumental dans lequel la fondation de la nouvelle cité de Cyzique par une autre population est censée prendre place. C'est ce paysage, et les rites qui l'habitent, et non une forme de descendance ou de parenté, qui font le lien entre les Dolions disparus (du moins au sens culturel et politique) et les Ioniens de la Cyzique historique. Cette articulation donne toute sa cohérence au récit d'Apollonios. Celui-ci peut bien différer sur certains points des versions antérieures qui ont organisé autrement le matériau légendaire (Vian 1974, p.30-33 et 1982). Ces différences ne font que mettre en lumière la logique de la narration, qui est celle du passage brutal d'un monde proche de l'âge d'or à un monde pleinement inscrit dans l'histoire. Pour les raisons que nous avons mentionnées, l'introduction par Apollonios de Poséidon dans ce bouleversement ne peut être considérée comme un simple «élément rapporté » (Vian 1974, p. 30-34) sans lien clair avec le reste du récit. Mais doit-on pour autant considérer que le dieu, comme cela est si souvent écrit, se trouve dans la position de divinité vaincue, dépossédée, parce que ses protégés ont été anéantis? L'existence à Cyzique d'un culte de Poséidon Asphaleios («qui assure, fortifie ») (Thély 2014) ne suffit pas à répondre à cette question. Il convient, pour cela, de se tourner vers d'autres situations où le dieu est associé à la disparition d'un monde en suspens.

7 Tel pourrait être le cas des Méropes, le premier peuple à vivre sur l'île de Cos alors appelée Méropè ou Méropis. Tout en étant beaucoup plus succints que l'histoire des Dolions, les récits sur leur fin comportent certains éléments qui autorisent le rapprochement avec ces derniers. Ainsi, le roi des Méropes, Eurypyle (Iliade, II, 677), est présenté tantôt comme un "gègénès " tantôt comme un fils de Poséidon (Etienne de Byzance, s.v. Côs ; ps-Apollodore, Bibliothèque II, 7, 1). L'agent extérieur qui va apporter la destruction est Héraclès. On a vu comment celui-ci intervient en première ligne dans le combat des Argonautes contre les géants de Cyzique, où il semble se conformer à son rôle classique de destructeur de monstres. Or, son arrivée à Cos n'est pas sans analogie avec le retour malencontreux des Argonautes chez les Dolions. En effet, après avoir capturé Troie avec Télamon pour se venger du roi Laomédon et s'emparer de ses chevaux divins, Héraclès s'embarque pour revenir en Grèce; mais, pris dans une 
tempête déclenchée par Héra, il est dérouté vers Cos (Iliade XIV, 249-255). Là, son arrivée inopinée est prise pour un débarquement de pirates : voulant le repousser, les Méropes lui jettent des pierres, une façon bien connue de tenir un navire à distance et d'empêcher la descente à terre de son équipage. Néanmoins Héraclès parvient à forcer le passage, tue Eurypyle et dévaste ensuite l'île (ps-Apollodore, loc. cit.). Les Questions grecques de Plutarque ( $Q G 58$ ) livrent un récit un peu différent mais plus détaillé qui sert d'aition à un trait particulier du culte d'Héraclès à Cos (Paul 2013, p. 251-252). Pour expliquer le fait que le prêtre d'Héraclès dans le dème d'Antimachia s'habille en femme pour sacrifier au dieu, Plutarque raconte qu'après avoir débarqué à une extrémité de l'île, Héraclès rencontre un berger et lui demande un bélier (autre thème récurrent des parcours du héros affamé) ; se heurtant au refus du berger, il le défie à la lutte mais, face à l'afflux des Méropes venus prêter main forte à son adversaire, il est contraint de se réfugier chez une femme thrace et de se déguiser en femme. C'est sur le lieu même de ce combat que se perpétue le sacrifice à Héraclès. Dans un deuxième temps, celui-ci triomphe des Méropes. Le récit de Plutarque est évidemment riche en connotations propres aux légendes et aux cultes d'Héraclès mais ce n'est pas l'aspect qui nous retiendra ici. On soulignera plutôt, que dans cet épisode aussi, l'arrivée d'un héros détourné de sa route par une tempête aboutit à la destruction de la population locale. La tempête est l'élément qui, en interrompant la linéarité de l'action, permet la découverte inattendue d'une île «inconnue» (apparemment inconnue pour les Argonautes; réellement inconnue pour Héraclès). La soudaineté de cette arrivée déclenche une réaction hostile qui est punie par la dévastation de l'île, et celle-ci à son tour ouvre la voie à la naissance d'une cité historique, en l'occurrence la Cos fondée par les Doriens d'Épidaure et Trézène. Comme à Cyzique, l'intervention du héros venu de l'extérieur fait basculer l'île dans l'histoire, d'une part en faisant disparaître la population primitive - parfois rattachée aux «nés de la terre »-, d'autre part, en créant le paysage cultuel qui est supposé fournir le cadre de la création de la cité historique - dans ce cas les cultes d'Héraclès bien attestés à Cos (Paul 2013, p. 95-116). Assurément, Poséidon est ici moins directement présent que chez Apollonios. Mais la paternité du roi Eurypyle qui lui est parfois attribuée pourrait sembler le placer à nouveau dans la position de dieu vaincu par le héros "civilisateur ». On ne saurait cependant s'en tenir à une opposition aussi sommaire. Pratiquement toutes les sources font suivre l'extermination des Méropes de la participation d'Héraclès à la Gigantomachie, où le héros s'illustre particulièrement en combattant le "champion » de tous les Géants, le Géant au superlatif Alcyonée (e.g. Pindare Ném. IV, 25-30; Isthm. VI, 30-35). Héraclès se retrouve ainsi aux côtés de Poséidon qui, de son côté, poursuit le Géant Polybotès jusqu'à Cos, où il arrache un morceau de l'île pour le jeter sur son adversaire et l'enfouit ainsi sous ce qui devient l'îlot de Nisyros (Strabon, X, 5, 16). Il ne peut donc être question d'antagonisme entre un dieu défenseur des êtres primordiaux et de forces sauvages et un héros porteur des valeurs d'un monde nouveau. Mais pour comprendre quel rôle Poséidon joue dans le processus de basculement vers l'histoire des milieux insulaires, isolés et préservés, il convient de se tourner vers l'épisode qui peut être considéré comme le prototype des récits que je viens d'examiner et qui en illustre les dynamiques de manière paradigmatique : l'arrivée d'Ulysse dans l'île des Phéaciens dans l'Odyssée.

8 Le monde des Phéaciens est suffisamment connu pour qu'une présentation d'ensemble soit superflue. Il est le passage, la transition qui permet à Ulysse de quitter le monde des dieux, des nymphes et de toutes les créatures non humaines parmi lesquels il a 
erré, avant de regagner le monde des humains. C'est chez les Phéaciens que le héros délivre tout le récit de ses aventures dans le monde d'au-delà de l'humanité, comme si l'évocation de ce monde qu'il laisse derrière lui était le préalable nécessaire à son retour dans l'univers familier des «mangeurs de pain». Il s'agit donc bien d'une frontière, d'un passage «à la croisée des espaces » entre deux univers du récit, celui-là même où se met en place, par le jeu des oppositions fondamentales, une anthropologie propre à l'homme grec (Hartog 1996, p. 39-42). Or, ce seuil présente plusieurs traits qui intéressent directement notre propos. Il s'agit en premier lieu d'un monde placé sous la protection directe de Poséidon : le dieu est le père de Nausithoos, fondateur de la cité sur l'île de Schérie et père d'Alcathoos (Homère, Odyssée, VII, 56-57). Son temple domine l'agora entre les deux ports (Od. VI, 266-267). Par certains aspects Schérie présente les traits d'une véritable cité avec son agora, son temple, ses remparts et ses institutions (Mossé 1980); les Phéaciens peuvent même pratiquer piraterie et enlèvements (l'intendante Eurymedousa, enlevée d'Apéirè et offerte à Alkinoos : Od. VII, 7-12). Mais elle appartient simultanément à un monde au voisinage immédiat des dieux et des êtres primordiaux. Périboia, avec qui Poséidon avait engendré Nausithoos, avait pour père Eurymédon, «qui jadis régnait sur les Géants orgueilleux » (Od. VII, 58-59). Avant que Nausithoos ne les installe à Schérie, les Phéaciens vivaient à Hypérée, dans une situation qui n'est pas sans préfigurer celle des Dolions d'Apollonios vivant au voisinage des «nés de la terre » (Od. VI, 4-6) :

Jadis ils habitaient dans la spacieuse Hypérée, près des Cyclopes arrogants dont ils devaient subir la force et les pillages.

Par ailleurs, quand il parle des dieux, Alkinoos déclare à Ulysse (Od. VII, 202-206) :

Quand nous leur offrons de glorieuses hécatombes, ils viennent au festin s'asseoir à nos côtés, aux mêmes bancs que nous ; sur le chemin désert, s'ils croisent l'un des nôtres, ils ne se cachent pas car nous leur sommes apparentés, tout comme les Cyclopes et les tribus sauvages des Géants.

10 Se présentant comme une cité humaine pourvue de traits historiques, l'univers des Phéaciens appartient tout autant à un âge d'or où les hommes côtoient les dieux et les êtres primordiaux et partagent leurs festins avec eux. Ce double visage correspond bien à une position de frontière, de monde à cheval entre les deux mondes, humain et nonhumain. Les Phéaciens vivent dans un temps qui n'est pas entièrement le temps historique; par bien des aspects, ils appartiennent à cet autre temps où hommes et dieux vivaient en connivence. Et ce caractère à part se traduit aussi par leur isolement spatial. Schérie en effet est inaccessible par les moyens ordinaires. Les Phéaciens sont des navigateurs réputés qui savent « donner le passage à tous »; ils ont reçu ce don de Poséidon (Od. VIII, 566 ; VII, 35). Mais ils n'auraient pas cette compétence s'ils ne disposaient pas, grâce au dieu, de navires bien singuliers, comme Alkinoos l'explique (Od. VIII, 560-563) :

Les Phéaciens n'ont ni pilotes, ni gouvernails comme sur les autres navires, mais nos navires connaissent les pensées et les esprits des hommes, connaissent les cités et les riches campagnes de tous les peuples, et passent au-dessus du gouffre des mers dissimulés dans la brume et les nuées ; jamais ils ne craignent ni l'avarie ni la ruine.

11 Ce n'est donc ni la science ni l'expérience des Phéaciens qui leur permet d'aller où ils veulent sur l'étendue marine; ils n'ont même pas de kubernètèr. D'ailleurs leurs traversées s'effectuent " dans la brume et les nuées ", donc sans aucun point de repère et c'est de nuit que s'effectue le voyage vers Ithaque. Les traversées phéaciennes 
ressemblent plutôt à des passages entre deux mondes bien distincts qu'aucun moyen ordinaire ne permet de relier, dans l'obscurité nécessaire à tout changement non pas de lieu, mais de dimension.

De même, c'est de manière bien singulière qu'Ulysse arrive sur les rivages phéaciens. Alors que le héros a navigué sans encombre sur son radeau pendant dix-sept jours, c'est au moment où il arrive tout près de l'île $(O d . \mathrm{V}, 280)$ que Poséidon, tout juste de retour du pays des Éthiopiens, l'aperçoit et déclenche une tempête " pour accroître sa charge de malheur" (Od. V, 290). Rejeté au large, Ulysse voit apparaître la déesse InoLeucothée qui lui donne son voile, et sur ce voile Ulysse nage deux jours dans la mer agitée avant de pouvoir enfin gagner le rivage de Schérie, non sans mal (Od. V, 333-462). L'épisode n'est pas sans paradoxe. Nulle part il n'est dit que Poséidon veuille empêcher Ulysse d'atteindre Schérie : le dieu se contente de répéter qu'il veut accabler le héros de malheurs. La colère de Poséidon, motivée par l'offense faite à son fils Polyphème, n'est pas aussi absolue et continue qu'on le pense généralement: l'épisode de la tempête devant Schérie est en fait le seul cas où Poséidon se déchaîne vraiment contre le héros (Murgatroyd 2015). Le dieu en fait ne peut pas faire obstacle au retour d'Ulysse, voulu par les dieux, mais il l'empêche de revenir dans les conditions que le héros aurait souhaitées. De plus, c'est l'intervention d'une divinité marine bien connue, qui ne saurait aller directement à l'encontre des volontés de son puissant seigneur, qui permet à Ulysse de gagner la terre. Il n'y a donc pas contradiction entre la colère de Poséidon et l'acte salvateur de Leucothée : il convient plutôt de les voir comme complémentaires. Poséidon ne peut pas ne pas se mettre en colère contre Ulysse ; mais, dans la logique du récit, l'arrivée d'Ulysse chez les Phéaciens ne peut pas être définitivement empêchée par cette colère. Et en aucun cas Poséidon ne peut paraître humilié par cette arrivée. Est-ce à dire que l'épisode ne ferait que retarder le débarquement d'Ulysse chez les Phéaciens? Ce serait peu de chose pour un récit aussi long. Peut-être y-a-t-il une autre signification à tout ce récit : la double intervention de Poséidon et de Leucothée, loin d'être contradictoire, est articulée de manière à empêcher Ulysse d'arriver à Schérie au terme d'une navigation normale, par ses propres moyens. L'aide d'une divinité est nécessaire pour qu'il puisse atteindre son but. Autrement dit, pas plus que ses habitants ne quittent leur île avec des navires normaux ordinaires, personne ne peut arriver en Phéacie par des moyens normaux. L'île ne peut être atteinte sans l'aide des dieux - un thème qu'utilisent aussi, à leur façon, les récits sur les Dolions et les Méropes avec leurs tempêtes respectives.

13 L'île des Phéaciens est donc bien hors du temps et de l'espace ordinaires; il s'agit, là encore, d'un «monde en suspens" protégé par Poséidon. L'arrivée d'Ulysse est l'élément extérieur qui va précipiter la fin de cet univers à part. Non pas, évidemment, parce qu'Ulysse s'en prend directement aux Phéaciens ; mais parce que, en ramenant le héros à Ithaque, les Phéaciens perdent la protection que Poséidon leur accordait jusqu'alors. En pétrifiant leur navire qui rentre au port après avoir fait le voyage d'Ithaque (Od. XIII, 159-164), le dieu signifie aux Phéaciens que le temps de leur statut à part, de leur âge d'or, est révolu, et qu'ils doivent désormais renoncer à leur qualité de passeurs infaillibles (Od. XIII, 172-182). Là aussi, l'arrivée du héros extérieur marque le moment où l'île bascule dans le temps et dans l'espace ordinaires, où elle perd l'équilibre antérieur et la connivence entre hommes et dieux pour entrer dans l'histoire purement et simplement humaine. Mais on relève que dans ce cas c'est Poséidon luimême, tout protecteur des Phéaciens qu'il ait été, qui les précipite hors de leur âge 
d'or. La perte, le changement que subissent les Phéaciens n'est, de ce fait, en rien une atteinte à la souveraineté et à la puissance du dieu. Le dieu lui-même décide que le moment est venu de mettre fin à cette exception que représente Schérie, et de l'intégrer tout entière dans le monde des hommes. Poséidon, loin d'être une victime des changements qui mettent fin à la proximité des hommes avec les dieux ou les géants, doit être perçu comme un des acteurs de ces transformations qui sont nécessaires pour faire basculer le récit dans l'histoire. C'est une raison de plus pour en finir avec une vision de Poséidon comme dieu déclassé, vaincu, un dieu du passé dont le rôle est amoindri dans la Grèce historique ${ }^{6}$. Que le basculement dans l'histoire des mondes en suspens s'opère brutalement, dans la violence, ne saurait surprendre : ce n'est pas le propre de Poséidon d'agir dans la douceur, le dieu opère par secousses et ruptures brutales. Mieux même, on peut comprendre ce rôle du dieu en le rapprochant de sa fonction d'« ébranleur » : certes, l'ébranlement en question est avant tout tellurique, Poséidon agit en " ébranleur du sol », Ennosigaios ou Enosichtôn. Mais pourquoi ébranler, mettre en mouvement (dans le sens de "mettre en branle»), ne pourrait s'appliquer aussi au temps, ou plutôt à cette combinaison particulière d'espace et de temps " hors du monde » que représentent les îles « en suspens »? Poséidon apparaîtrait ainsi à la fois comme le protecteur de mondes clos et lointains où se perpétue une forme d'âge d'or, mais aussi comme celui qui, le moment venu, agit ou laisse agir pour que ces mondes opèrent le passage nécessaire et rapide vers l'histoire : une ambivalence tout à fait compréhensible pour ce dieu qui est tout à la fois l'un des plus puissants parmi les Olympiens et le plus proche de toutes les créatures monstrueuses issues des âges primordiaux.

\section{BIBLIOGRAPHIE}

Ampolo C. éd. (2009), Immagine e immagini della Sicilia e di altre isole del Mediterraneo antico, Pise.

André L.-N. (2010), « L'escale à Cyzique (Apollonios de Rhodes, Les Argonautiques I, 922-1152) : espaces phobiques, stéréotypes paysagers et transfiguration ", Rursus (en ligne) 5. URL : https:// journals.openedition.org/rursus/422.

Ceccarelli P. (2009), « Isola e terraferma : la percezione della terra abitata in Grecia arcaica e classica », dans Ampolo 2009, p. 31-50.

Cusset Ch. (2004), « Les Argonautiques d'Apollonios de Rhodes comme itinéraire à travers la sauvagerie : d'Homère à Alexandrie, en passant par Hérodote et Xénophon, ou comment l'adresse au lecteur supplée à l'insouciance de Jason ", dans Les espaces du sauvage dans le monde antique: approches et définitions. Actes du colloque (Besançon, 4-5 mai 2000), Besançon, p. 31-52.

Darthou S. (2005), « Retour à la terre : fin de la geste d'Érechtée », Kernos 18, p. 69-83.

Darthou S. (2008), «Éris dans la cité. Quelques réflexions sur les 'cosmogonies' politiques », Métis N.S.6, p. 269-285.

Doyen C. (2011), Poséidon souverain. Contribution à l'étude religieuse de la Grèce mycénienne et archaïque, Bruxelles. 
Hartog F. (1996), Mémoires d'Ulysse. Récits sur la frontière en Grèce ancienne, Paris.

Mossé C. (1980), « Ithaque ou la naissance de la cité », AION 2, p. 7-19.

Murgatroyd P. (2015), « The Wrath of Poseidon », The Classical Quaterly 65-2, p. 444-448.

Napoli J. éd. (2008), Ressources et activités maritimes des peuples de l'Antiquité, Actes du colloque international de Boulogne-sur-Mer, 12, 13 et 14 mai 2005, Les Cahiers du Littoral 2-6, Boulogne-surMer.

Paul S. (2013), Cultes et sanctuaires de l'île de Cos, Kernos Suppl. 28, Liège.

Pirenne-Delforge V., Pironti G. (2016), L’Héra de Zeus. Ennemie intime, épouse définitive, Paris.

Polignac F. de (2017), « Détroits, isthmes, passages : paysages “sous le joug” de Poséidon », Kernos 30, p. $67-83$.

Préteux F. (2008), « Le commerce de Cyzique au IV siècle », dans Napoli 2008, p. 353-368.

Schlosser P. (2014), « Cyzique et la mer », dans Sève et Schlosser 2014, p. 253-277.

Sève M. et Schlosser P. éd. (2014), Cyzique, cité majeure et méconnue de la Propontide antique, Metz.

Thély L. (2014), « Le culte de Poséidon Asphaleios à Cyzique », dans Sève et Schlosser 2014, p. 179-193.

Vian F. (1944), « Géants de la mer », RA 22, p. 97-117.

Vian F. (1952), « Génies des passes et des défilés », RA 39, p. 129-155.

Vian F. (1974), « Notice », dans Apollonios de Rhodes, Les Argonautiques, Paris, Les Belles Lettres, p. 1-49.

Vian F. (1982), « Les navigations des Argonautes. Élaboration d'une légende », BAGB, p. 273-285.

Vilatte S. (1991), L'insularité dans la pensée grecque, Besançon.

\section{NOTES}

1. Apollonios parle à la fois d'une île et d'un « isthme baigné par la mer » (v. 938), ce qui peut prêter à confusion. La presqu'île d'Arktonnèse, site de Cyzique, semble avoir été originellement une île, et c'est sans doute à partir de l'époque hellénistique qu'elle a été reliée au continent par un tombolo: Préteux 2008, p. 354-356; Schlosser 2014, p. 260-262. Mais le mot nèsos pourrait avoir été employé aussi bien pour des péninsules que pour des îles : Ceccarelli 2009, p. 34.

2. Mon analyse diffère sur ce point de celle de Christophe Cusset, qui ne voit dans les Gègénées que de simples «faire-valoir» de l'humanité des Dolions (Cusset 2004, p. 33-35).

3. Laury-Nuria André (André 2010) a bien montré la signification de cette menace d'enfermement dans l'économie générale de la topique insulaire. Voir aussi Vilatte 1991, p. 29-39, qui ne mentionne cependant pas le cas de Cyzique.

4. Sur l'importance des dédoublements d'actions avec une valeur tantôt positive tantôt négative dans le récit, voir André 2010.

5. Sur l'identification et la localisation des ports de Cyzique et des lieux mentionnés par Apollonios, voir Schlosser 2014, p. 262-267. 
6. Idée assez diffuse dans maints travaux sur la religion grecque; voir dernièrement Doyen 2011. À l'inverse, les travaux de Sonia Darthou (2005 et 2008) montrent le rôle du dieu dans la construction du politique.

\section{RÉSUMÉS}

Plusieurs légendes associent le dieu Poséidon à des milieux insulaires placés en marge du temps et de l'espace ordinaires de l'humanité. L'île des Dolions et des "Nés de la Terre » dans les Argonautiques d'Apollonios, l'île de Cos dans la légende des Méropes, mais aussi l'île des Phéaciens dans l'odyssée, sont quelques exemples de ces mondes clos, immobiles, où se maintiennent, hors de l'histoire et sous la protection de Poséidon, un équilibre particulier, une forme de connivence entre la vie des hommes et la présence d'entités non humaines, dieux, géants, cyclopes et autres êtres primordiaux. Dans tous ces cas, l'irruption d'un intervenant extérieur déclenche une rupture de l'ordre et de l'équilibre qui prévalaient jusque-là, met fin à la proximité avec les dieux ou les géants et fait basculer ces mondes dans un espace et un temps pleinement et exclusivement humains. Ces îles intègrent ainsi le cours d'une histoire dont elles étaient restées séparées. Mais cette rupture n'est pas une défaite de Poséidon : bien au contraire, le dieu semble parfois cautionner lui-même le processus de passage d'un monde en suspens au monde de l'histoire.

Several legends associate the god Poseidon with islands set on the margins of human space and time. The Dolions of Kysikus and their neighbours "Born of the Earth", Cos and the Merops, but also the island of Phaeacia in the Odyssey, are some examples of isolated and immutable worlds outside of history where Poseidon's protection maintains a fragile balance between human populations and the presence of gods, giants or other non-human creatures. In all these cases, the irruption of an external agent breaks this order and balance, puts an end to the proximity between human and non-human beings and forces these worlds to enter the purely human space and time. These islands then enter the normal course of history, from which they had remained separated until there. But this rupture is not a defeat for Poseidon: quite on the contrary, the god sometimes seems to protect this shift from immutability to history.

\section{INDEX}

Mots-clés : Poséidon, Cyzique, Dolions, Cos, Méropes, Phéacie, insularité, temporalité

Keywords : Poseidon, Kyzikus, Dolions, Cos, Merops, Pheacia, insularity, temporality

\section{AUTEUR}

\section{FRANÇOIS DE POLIGNAC}

EPHE-PSL, UMR 8210 ANHIMA 